\title{
COMMUTATIVE ALGEBRAS FOR ARRANGEMENTS
}

\author{
PETER ORLIK AND HIROAKI TERAO ${ }^{1}$
}

\section{Introduction}

Let $V$ be a vector space of dimension $l$ over some field $\mathbf{K}$. A hyperplane $H$ is a vector subspace of codimension one. An arrangement $\mathscr{A}$ is a finite collection of hyperplanes in $V$. We use [7] as a general reference. Let $M(\mathscr{A})=V-\cup_{H \in \mathscr{A}} H$ be the complement of the hyperplanes. Let $V^{*}$ be the dual space of $V$. Each hyperplane $H \in \mathscr{A}$ is the kernel of a linear form $\alpha_{H} \in V^{*}$, defined up to a constant. The product

$$
Q(\mathscr{A})=\prod_{H \in \mathscr{A}} \alpha_{H}
$$

is called a defining polynomial of $\mathscr{A}$. Brieskorn [3] associated to $\mathscr{A}$ the finite dimensional skew-commutative algebra $R(\mathscr{A})$ generated by 1 and the differential forms $d \alpha_{H} / \alpha_{H}$ for $H \in \mathscr{A}$. When $\mathbf{K}=\mathbf{C}$, the algebra $R(\mathscr{A})$ is isomorphic to the cohomology algebra of the open manifold $M(\mathscr{A})$. The structure of $R(\mathscr{A})$ was determined in [6] as the quotient of an exterior algebra by an ideal. In particular this shows that $R(\mathscr{A})$ depends only on the intersection poset of $\mathscr{A}, L(\mathscr{A})$, and not on the individual linear forms $\alpha_{H}$.

A subarrangement $\mathscr{B} \subseteq \mathscr{A}$ is called independent if $\cap_{H \in \mathscr{B}} H$ has codimension $|\mathscr{B}|$, the cardinality of $\mathscr{B}$. In a special lecture at the Japan Mathematical Society in 1992, Aomoto suggested the study of the graded $\mathbf{K}$-vector space

$$
A O(\mathscr{A})=\sum_{\mathscr{B}} \mathbf{K} Q(\mathscr{B})^{-1}, \quad \mathscr{B} \text { independent. }
$$

It appears as the top cohomology group of a certain 'twisted' de Rham chain complex [1]. When $\mathbf{K}=\mathbf{R}$, he conjectured that the dimension of $A O(\mathscr{A})$ is equal to the number of connected components (chambers) of $M(\mathscr{A})$, which he proved for generic arrangements. In this paper we prove this conjecture in general. We construct a

Received March 3, 1993.

1 This work was supported in part by the National Science Foundation 
commutative algebra $W(\mathscr{A})$ which is isomorphic to $A O(\mathscr{A})$ as a graded vector space. Note that $A O(\mathscr{A})$ is not closed under multiplication because a product $Q(\mathscr{B})^{-1} Q\left(\mathscr{B}^{\prime}\right)^{-1}$ may contain the same linear form $\alpha_{H}$ more than once. In order to allow this, we need the following definition. A multiarrangement $\mathscr{E}$ is a finite set of hyperplanes where each hyperplane may occur more than once. The multiplicity of $H$ in $\mathscr{E}, m(H, \mathscr{E})$ is the number of times $H$ occurs in $\mathscr{E}$. The cardinality of $\mathscr{E},|\mathscr{E}|$, is the total number of elements of $\mathscr{E}$, each hyperplane counted with its multiplicity. Let $\mathbf{E}_{p}(\mathscr{A})$ be the set of multisubarrangements $\mathscr{E}$ of $\mathscr{A}$ of cardinality $p$. Let $\mathbf{E}(\mathscr{A})=\cup_{p \geq 0} \mathbf{E}_{p}(\mathscr{A})$. This union is disjoint. We write $\mathbf{E}=\mathbf{E}(\mathscr{A})$ when $\mathscr{A}$ is fixed. Let $\cap \mathscr{E}=\cap_{H \in \mathscr{E}} H$. We call $\mathscr{E} \in \mathbf{E}$ independent if $\operatorname{codim}(\cup \mathscr{E})=|\mathscr{E}|$, and dependent otherwise. Note that if $m(H, \mathscr{E})>1$ for some $H \in \mathscr{E}$, then $\mathscr{E}$ is dependent. Let $\mathbf{E}^{i}$ denote the set of independent multisubarrangements. This is a finite set. Let $\mathbf{E}^{d}$ denote the set of dependent multisubarrangements. This is an infinite set. There is a disjoint union $\mathbf{E}=\mathbf{E}^{i} \cup \mathbf{E}^{d}$. Let $S=S\left(V^{*}\right)$ be the symmetric algebra of $V^{*}$. Choose a basis $\left\{e_{1}, \ldots, e_{l}\right\}$ in $V$ and let $\left\{x_{1}, \ldots, x_{l}\right\}$ be the dual basis in $V^{*}$ so $x_{i}\left(e_{j}\right)=\delta_{i, j}$. We may identify $S\left(V^{*}\right)$ with the polynomial algebra $S=\mathbf{K}\left[x_{1}, \ldots, x_{l}\right]$. Let $Q(\mathscr{E})=\Pi_{H \in \mathscr{E}} \alpha_{H}$ for $\mathscr{E} \in \mathbf{E}$. Note that $\alpha_{H}$ appears with multiplicity $m(H, \mathscr{E})$ in $Q(\mathscr{E})$. Let $S_{(0)}$ be the field of quotients of $S$, the field of rational functions on $V$.

Definition 1.1. Let $\mathbf{K}\left[\alpha_{\mathscr{A}}^{-1}\right]$ be the $\mathbf{K}$-subalgebra of $S_{(0)}$ generated by

$$
\left\{Q(\mathscr{E})^{-1} \mid \mathscr{E} \in \mathbf{E}\right\} .
$$

Let $J(\mathscr{A})$ be the ideal of $\mathbf{K}\left[\alpha_{\mathscr{A}}^{-1}\right]$ generated by $\left\{Q(\mathscr{E})^{-1} \mid \mathscr{E} \in \mathbf{E}^{d}\right\}$.

Let $W(\mathscr{A})=\mathbf{K}\left[\alpha_{\mathscr{A}}^{-1}\right] / J(\mathscr{A})$.

Consider the usual grading of $S_{(0)}$. Since $J(\mathscr{A})$ is a homogeneous ideal, $W(\mathscr{A})$ is a graded commutative algebra. There is a natural map of graded vector spaces $j: A O(\mathscr{A}) \rightarrow W(\mathscr{A})$ defined by $Q(\mathscr{B})^{-1} \mapsto\left[Q(\mathscr{B})^{-1}\right]$. It is clear that $A O(\mathscr{A})$ is finite dimensional because the set $\mathbf{E}^{i}$ is finite. Since the map $j$ is surjective, the algebra $W(\mathscr{A})$ is also a finite dimensional $\mathbf{K}$-vector space. Its total dimension, Poincare polynomial, or algebra structure are not obvious at this point. In the rest of this paper we determine these.

In Section 2 we define a polynomial algebra $\mathbf{K}\left[u_{\mathscr{A}}\right]$ based on $\mathscr{A}$ and a quotient algebra $U(\mathscr{A})$. We also study some properties of $U(\mathscr{A})$. Section 3 contains the proof that $U(\mathscr{A})$ and $W(\mathscr{A})$ are isomorphic graded algebras. In Section 4 we compute the Poincaré polynomial of $W(\mathscr{A})$ and prove that $j$ is an isomorphism of vector spaces. 
It is not clear from our results whether $W(\mathscr{A})$ depends only on $L(\mathscr{A})$ or not. Another interesting question is if $W(\mathscr{A})$ is the model for any topological invariant of $M(\mathscr{A})$.

\section{The algebra $U(\mathscr{A})$}

Let $\mathscr{A}$ be an arrangement. Let $L=L(\mathscr{A})$ be the set of all intersections of elements of $\mathscr{A}$. We agree that $L$ includes $V$ as the intersection of the empty collection of hyperplanes. We should remember that if $X \in L$, then $X \subseteq V$. Partially order $L$ by reverse inclusion. Then $L$ is a geometric lattice with rank function $r(X)=$ $\operatorname{codim}(X)$ [7, Lemma 2.3].

Let $\mathbf{E}_{X}=\{\mathscr{E} \in \mathbf{E} \mid \cap \mathscr{E}=X\}$. Then we have the disjoint union

$$
\mathbf{E}=\cup_{X \in L} \mathbf{E}_{X}
$$

We use notation such as $\mathbf{E}_{p, X}=\mathbf{E}_{p} \cap \mathbf{E}_{X}, \mathbf{E}_{p, X}^{\imath}=\mathbf{E}^{\imath} \cap \mathbf{E}_{p, X}$, etc.

Definition 2.1. Let $\mathbf{K}\left[u_{\mathscr{A}}\right]$ be the polynomial ring in the indeterminates $u_{H}, H \in \mathscr{A}$. Write $u_{\mathscr{g}}=\Pi_{H \in \mathscr{E}} u_{H}$. Define

$$
\begin{array}{ll}
\mathbf{K}\left[u_{\mathscr{A}}\right]_{p}=\sum_{\mathscr{E} \in \mathbf{E}_{p}} \mathbf{K} u_{\mathscr{E}}, & \mathbf{K}\left[u_{\mathscr{A}}\right]_{X}=\sum_{\mathscr{E} \in \mathbf{E}_{X}} \mathbf{K} u_{\mathscr{E}}, \\
\mathbf{K}\left[u_{\mathscr{A}}\right]^{i}=\sum_{\mathscr{E} \in \mathbf{E}^{\prime}} \mathbf{K} u_{\mathscr{E}}, & \mathbf{K}\left[u_{\mathscr{A}}\right]^{d}=\sum_{\mathscr{E} \in \mathbf{E}^{d}} \mathbf{K} u_{\mathscr{E}} .
\end{array}
$$

We have the following direct sum decompositions:

$$
\begin{aligned}
& \mathbf{K}\left[u_{\mathscr{A}}\right]=\oplus_{p \geq 0} \mathbf{K}\left[u_{\mathscr{A}}\right]_{p}, \\
& \mathbf{K}\left[u_{\mathscr{A}}\right]=\oplus_{X \in L} \mathbf{K}\left[u_{\mathscr{A}}\right]_{X}, \\
& \mathbf{K}\left[u_{\mathscr{A}}\right]=\mathbf{K}\left[u_{\mathscr{A}}\right]^{i} \oplus \mathbf{K}\left[u_{\mathscr{A}}\right]^{d} .
\end{aligned}
$$

Let $\pi_{p}, \pi_{X}, \pi^{i}$, and $\pi^{d}$ be the respective projections. These maps commute pairwise. We use notation such as $\mathbf{K}\left[u_{\mathscr{A}}\right]_{p, X}=\mathbf{K}\left[u_{\mathscr{A}}\right]_{p} \cap \mathbf{K}\left[u_{\mathscr{A}}\right]_{X}, \mathbf{K}\left[u_{\mathscr{A}}\right]_{p, X}^{\imath}=$ $\mathbf{K}\left[u_{\mathscr{A}}\right]^{\imath} \cap \mathbf{K}\left[u_{\mathscr{A}}\right]_{p, X}$, etc.

Definition 2.2. Let $I(\mathscr{A})$ be the ideal of $\mathbf{K}\left[u_{\mathscr{A}}\right]$ generated by

(i) the elements of $\mathbf{K}\left[u_{\mathscr{A}}\right]^{d}$,

(ii) when $\sum_{H \in \mathscr{E}} c_{H} \alpha_{H}=0$ with $c_{H} \in \mathbf{K}$, the element $\sum_{H \in \mathscr{E}} c_{H} u_{\mathscr{g}-\{H\}}$.

Let $U(\mathscr{A})=\mathbf{K}\left[u_{\mathscr{A}}\right] / I(\mathscr{A})$. 
Grade $\mathbf{K}\left[u_{\mathscr{A}}\right]$ by $\operatorname{deg} u_{H}=-1$. Since $I(\mathscr{A})$ is a homogeneous ideal, $U(\mathscr{A})$ is a graded commutative $\mathbf{K}$-algebra. The isomorphism $U(\mathscr{A}) \simeq \mathbf{K}\left[u_{\mathscr{A}}\right]^{i} / I(\mathscr{A}) \cap$ $\mathbf{K}\left[u_{\mathscr{A}}\right]^{i}$ shows that $U(\mathscr{A})$ is a finite dimensional graded commutative $\mathbf{K}$-algebra.

A circuit $C=\left\{H_{1}, \ldots, H_{k}\right\}$ is a minimally dependent subset of hyperplanes: $C$ is dependent, but $C-\left\{H_{i}\right\}$ is independent for all $i$.

PRoposition 2.3. The ideal $I(\mathscr{A})$ is generated by the following finite set:

(a) $u_{H}^{2}$ for $H \in \mathscr{A}$,

(b) for each circuit $C=\left\{H_{1}, \ldots, H_{k}\right\}$ with $\sum_{i=1}^{k} c_{i} \alpha_{H_{i}}=0$, the element $\sum_{i=1}^{k} c_{i} u_{C-H_{i}}$.

Proof. Let $I^{\prime}$ denote the ideal generated by elements of type (a) and (b) of the proposition. It is clear that $I^{\prime} \subseteq I$. To prove the converse, we argue separately for elements of type (i) and (ii) of the definition.

(i) Suppose $f \in \mathbf{K}\left[u_{\mathscr{A}}\right]^{d}$. Since $\mathbf{K}\left[u_{\mathscr{A}}\right]^{d}$ is an ideal, it suffices to assume that $f=u_{c}$, where $C=\left\{H_{1}, \ldots, H_{k}\right\}$ is a circuit. Suppose $\sum_{i=1}^{k} c_{i} \alpha_{H_{i}}=0$. It follows from (b) that $\sum_{i=1}^{k} c_{i} u_{C-H_{i}} \in I^{\prime}$ and $u_{H_{1}} \sum_{i=1}^{k} c_{i} u_{C-H_{i}} \in I^{\prime}$. We use the distributive law and (a) to conclude that $c_{1} u_{C} \in I^{\prime}$. Since $C$ is a circuit, $c_{1} \neq 0$. Thus $f \in I^{\prime}$.

(ii) We show that for each relation $\sum_{H \in \mathscr{B}} c_{H} \alpha_{H}=0$ with $c_{H} \in \mathbf{K}$, the corresponding element $\sum_{H \in \mathscr{E}} c_{H} u_{\mathscr{E}-\{H\}} \in I^{\prime}$. Suppose not. Choose a counterexample with minimal $|\mathscr{E}|$. Note that minimality implies that for every $H \in \mathscr{E}, m(H, \mathscr{E})=1$. Let $\mathscr{E}=\left\{H_{1}, \ldots, H_{m}\right\}$ with distinct $H_{j}$. Let $\sum_{i=1}^{m} c_{i} \alpha_{H_{i}}=0$ be the corresponding relation. Since $\mathscr{E}$ is dependent, it contains a circuit. We may assume that $C=\left\{H_{1}\right.$, $\left.\ldots, H_{k}\right\}, k \leq m$, is a circuit. Thus $\sum_{i=1}^{k} a_{i} \alpha_{H_{i}}=0$ and $a_{i} \neq 0$ for $1 \leq i \leq k$. Define $a_{i}=0$ for $k+1 \leq i \leq m$. Then we have

$$
\sum_{i=1}^{m} c_{i} \alpha_{H_{i}}-\frac{c_{1}}{a_{1}} \sum_{i=1}^{k} a_{i} \alpha_{H_{i}}=\sum_{i=2}^{m}\left(c_{i}-\frac{c_{1}}{a_{1}} a_{i}\right) a_{H_{i}}=0 .
$$

The index set of the last relation is $\mathscr{E}-\left\{H_{1}\right\}$. It follows from the minimality assumption, that the corresponding element

$$
\sum_{i=2}^{m}\left(c_{i}-\frac{c_{1}}{a_{1}} a_{i}\right) u_{\mathscr{E}-\left\{H_{1}, H_{i}\right\}} \in I^{\prime}
$$

Multiply by $u_{H_{1}}$ and rewrite to get

$$
\sum_{i=1}^{m} c_{i} u_{\mathscr{g}-\left\{H_{i}\right\}}-\frac{c_{1}}{a_{1}} u_{\mathscr{E}-c} \sum_{i=1}^{k} a_{i} u_{C-\left\{H_{i}\right\}} \in I^{\prime}
$$

Since the second sum is in $I^{\prime}$ by (b), so is the first sum. This contradiction completes the argument. 


\section{The isomorphism}

Definition 3.1. Let $\Phi_{0}: \mathbf{K}\left[u_{\mathscr{A}}\right] \rightarrow S_{(0)}$ be the $\mathbf{K}$-algebra homomorphism induced by $u_{H} \mapsto \alpha_{H}^{-1}$. Since $\operatorname{im}\left(\Phi_{0}\right)=\mathbf{K}\left[\alpha_{\mathscr{A}}^{-1}\right]$, we have a surjective graded algebra homomorphism

$$
\Phi: \mathbf{K}\left[u_{\mathscr{A}}\right] \rightarrow \mathbf{K}\left[\alpha_{\mathscr{A}}^{-1}\right]
$$

Let $K=\operatorname{ker}(\Phi)$.

Lemma 3.2. (1) $\pi_{p}(K) \subseteq K$,

(2) $\pi_{X}(K) \subseteq K$,

(3) $\pi^{i}(K) \subseteq K$,

(4) $\pi^{d}(K) \subseteq K$.

Proof. (1) If a $\mathbf{K}$-linear combination of $Q(\mathscr{E})^{-1}$ is zero, then each homogeneous component of it is zero.

(2) Fix $f \in K$. By (1), we may assume that $f \in \mathbf{K}\left[u_{\mathscr{A}}\right]_{p}$. Write

$$
f=\sum_{\mathscr{E} \in \mathbf{E}_{p}} c_{\mathscr{E}} u_{\mathscr{E}}
$$

Let $f_{Z}=\pi_{Z}(f)$ for $Z \in L$. If $f_{Z} \in K$ for all $Z$, we are done. Suppose there exist some $Z \in L$ with $f_{Z} \notin K$. Among these $Z$ we choose one with minimal rank and call it $X$. Thus we may assume $f_{Y} \in K$ for all $Y$ with $r(Y)<r(X)$. We may write $\Phi(f)=0$ as

$$
\sum_{\substack{\mathscr{E} \in \mathbf{E}_{p} \\ n \mathscr{B}=X}} c_{\mathscr{E}} Q(\mathscr{\mathscr { E }})^{-1}=-\sum_{\substack{Y \neq X \\ f_{Y} \notin K}} \sum_{\substack{\mathscr{E} \in \mathbf{E}_{p} \\ n \mathscr{B}=Y}} c_{\mathscr{E}} Q(\mathscr{\mathscr { E }})^{-1} .
$$

Multiply both sides of (ii) by $Q(\mathscr{A})^{p}$. All the resulting terms are in $S$. We count zeros on both sides separately. We may choose coordinates so that $X=\left\{x_{1}=\cdots\right.$ $\left.=x_{r}=0\right\}$. Let $M$ be the ideal of $S$ generated by $\left\{x_{1}, \ldots, x_{r}\right\}$.

Let $c_{\mathscr{E}} Q(\mathscr{E})^{-1}$ be a term from the right side of (ii). Let $Y=\cap \mathscr{E} \neq X$. Note that $Y \varangle X$ because if $Y<X$, then $r(Y)<r(X)$ so $f_{Y} \in K$ by the minimality assumption. Thus $\mathscr{E} \cap\left(\mathscr{A}-\mathscr{A}_{X}\right) \neq \emptyset$. It follows that $Q(\mathscr{A})^{p} Q(\mathscr{E})^{-1} \in M^{p\left|\mathscr{A}_{X}\right|-p+1}$. Now consider the left side of (ii). Since $Q(\mathscr{A}) / Q\left(\mathscr{A}_{X}\right) \notin M$ and $M^{p\left|\mathscr{A}_{X}\right|-p+1}$ is an $M$-primary ideal, we have

$$
Q\left(\mathscr{A}_{X}\right)^{p} \sum_{\substack{\mathscr{E} \in \mathbf{E}_{p} \\ n \mathscr{E}=X}} c_{\mathscr{E}} Q(\mathscr{E})^{-1} \in M^{p\left|\mathscr{A}_{X}\right|-p+1}
$$


The degree of this nonzero polynomial is equal to $p\left|\mathscr{A}_{X}\right|-p$. This contradiction completes the argument.

(3) Suppose $f \in K$. It follows from (1) and (2) that we may assume $f \in$ $\mathbf{K}\left[u_{\mathscr{A}}\right]_{p, X}$. If $p>r(X)$, then $f \in \mathbf{K}\left[u_{\mathscr{A}}\right]^{d}$. Thus $\pi^{i} f=0 \in K$. If $p=r(X)$, then $f \in$ $\mathbf{K}\left[u_{\mathscr{A}}\right]^{i}$. Thus $\pi^{i} f=f \in K$.

(4) follows from (3) because $\pi^{i}+\pi^{d}=1$

TheOREM 3.3. The map $\Phi: \mathbf{K}\left[u_{\mathscr{A}}\right] \rightarrow \mathbf{K}\left[\alpha_{\mathscr{A}}^{-1}\right]$ induces an isomorphism of graded algebras $\phi: U(\mathscr{A}) \rightarrow W(\mathscr{A})$.

Proof. Since $\operatorname{ker}(\phi)=K+\mathbf{K}\left[u_{\mathscr{A}}\right]^{d}$, it is enough to show that $I=K+$ $\mathbf{K}\left[u_{\mathscr{A}}\right]^{\mathrm{d}}$. It is clear that $I \subseteq K+\mathbf{K}\left[u_{\mathscr{A}}\right]^{d}$ because the generators of $I$ of the second kind belong to $K$. Since $\mathbf{K}\left[u_{\mathscr{A}}\right]^{d} \subseteq I$, it suffices to show for the converse that if $f \in K \cap \mathbf{K}\left[u_{\mathscr{A}}\right]^{i}$, then $f \in I$. It follows from Lemma 3.2 (1) and (2) that we may assume $f \in K \cap \mathbf{K}\left[u_{\mathscr{A}}\right]_{p, X}^{i}$. We argue by induction on $p$. If $p=0$, then $f=0 \in I$. If $p>0$, then $\mathscr{A}_{X} \neq \emptyset$. Let $H_{0} \in \mathscr{A}_{X}$. Write $f=\sum_{\mathscr{E} \in \mathbf{E}_{p, X}^{i}} c_{\mathscr{E}} u_{\mathscr{E}}$. If $\mathscr{E} \in \mathbf{E}_{p, X}^{i}$, then $\left\{\mathscr{E}, H_{0}\right\}$ is a dependent set. Thus we have

$$
c_{0} \alpha_{H_{0}}+\sum_{H \in \mathscr{E}} c_{H} \alpha_{H}=0 .
$$

Since $\mathscr{E}$ is independent, $c_{0} \neq 0$ and we may assume that $c_{0}=1$. By definition we have

$$
u_{\mathscr{E}}+u_{H_{0}} \sum_{H \in \mathscr{E}} c_{H} u_{\mathscr{E}-\{H\}} \in K
$$

It follows that $f-u_{H_{0}} g \in K$ for $g \in \mathbf{K}\left[u_{\mathscr{A}}\right]_{p-1}^{i}$, so $u_{H_{0}} g \in K$. Since $K=$ $\operatorname{ker}(\Phi)$ is a prime ideal, $f \in K$ and $u_{H_{0}} \notin K$, we conclude that $g \in K$. Let $g_{Y}=$ $\pi_{Y}(g)$ and write $g=\sum g_{Y}$ where $g_{Y} \in \mathbf{K}\left[u_{\mathscr{A}}\right]_{p-1, Y}^{i}$. It follows from Lemma 3.2 (2) that $g_{Y} \in K$ for all $Y$. By the induction hypothesis, $g_{Y} \in I$. Thus $g \in I$ and $f \in I$.

\section{Structure theorems}

Let $U(\mathscr{A})_{X}=\mathbf{K}\left[u_{\mathscr{A}}\right]_{X} / \mathbf{K}\left[u_{\mathscr{A}}\right]_{X} \cap I$ and $W(\mathscr{A})_{X}=\mathbf{K}\left[\alpha_{\mathscr{A}}^{-1}\right]_{X} / \mathbf{K}\left[\alpha_{\mathscr{A}}^{-1}\right]_{X} \cap J$.

THEOREM 4.1. The algebra $U(\mathscr{A})$ is the direct sum $U(\mathscr{A})=\bigoplus_{X \in L} U(\mathscr{A})_{X}$.

Proof. It follows from Theorem 3.3 and Lemma 3.2 that $\pi_{X}(I)=\pi_{X}(K+$ $\left.\mathbf{K}\left[u_{\mathscr{A}}\right]^{d}\right) \subseteq K+\mathbf{K}\left[u_{\mathscr{A}}\right]^{d}=I$. It implies that $\pi_{X}(y) \in I \cap \mathbf{K}\left[u_{\mathscr{A}}\right]_{X}$ for any $y \in I$. 
Thus we have $I=\bigoplus_{X \in L}\left(I \cap \mathbf{K}\left[u_{\mathscr{A}}\right]_{X}\right)$. The result follows.

Theorem 4.2. The map $j: A O(\mathscr{A}) \rightarrow W(\mathscr{A})$ is an isomorphism of graded vector spaces.

Proof. Observe that $j$ is a graded, $\mathbf{K}$-linear, surjective map. It remains to show that $j$ is injective. Note that $A O(\mathscr{A})=\Phi\left(\mathbf{K}\left[u_{\mathscr{A}}\right]^{l}\right)$. Thus we have a commuting diagram.

$$
\begin{aligned}
& A O(\mathscr{A})=\Phi\left(\mathbf{K}\left[u_{\mathscr{A}}\right]^{i}\right) \simeq \mathbf{K}\left[u_{\mathscr{A}}\right]^{i} / K \cap \mathbf{K}\left[u_{\mathscr{A}}\right]^{1} \\
& j \downarrow \quad \downarrow \tau \\
& W(\mathscr{A}) \simeq U(\mathscr{A})=\mathbf{K}\left[u_{\mathscr{A}}\right] / I
\end{aligned}
$$

where $\tau$ is induced by the diagram. We show that $\tau$ is injective. It suffices to show that $I \cap \mathbf{K}\left[u_{\mathscr{A}}\right]^{i}=K \cap \mathbf{K}\left[u_{\mathscr{A}}\right]^{\imath}$. Theorem 3.3 implies that $I \cap \mathbf{K}\left[u_{\mathscr{A}}\right]^{\imath} \supseteq K \cap$ $\mathbf{K}\left[u_{\mathscr{A}}\right]^{2}$. For the converse, write an element of $I=K+\mathbf{K}\left[u_{\mathscr{A}}\right]^{d}$ as $a+b$, where $a$ $\in K$ and $b \in \mathbf{K}\left[u_{\mathscr{A}}\right]^{d}$. Then an arbitrary element of $I \cap \mathbf{K}\left[u_{\mathscr{A}}\right]^{i}$ is $\pi^{i}(a+b)=$ $\pi^{i}(a) \in K \cap \mathbf{K}\left[u_{\mathscr{A}}\right]^{2}$ by Lemma $3.2(2)$.

If $M=\oplus_{p \geq 0} M_{p}$ is a finite dimensional graded vector space, we let $\operatorname{Poin}(M, t)$ $=\sum_{p \geq 0}\left(\operatorname{dim} M_{p}\right) t^{p}$ be its Poincare polynomial. Recall $[7,2.42]$ the (one variable) Möbius function $\mu: L(\mathscr{A}) \rightarrow \mathbf{Z}$ defined by $\mu(V)=1$ and for $X>V$ by $\sum_{Y \leq X} \mu(Y)=0$.

\section{THEOREM 4.3.}

$$
\operatorname{Poin}(W(\mathscr{A}), t)=\operatorname{Poin}(U(\mathscr{A}), t)=\operatorname{Poin}(A O(\mathscr{A}), t)=\sum_{X \in L(\mathscr{A})} \mu(X)(-t)^{\tau(X)}
$$

Proof. It suffices to show that $\operatorname{dim} W(\mathscr{A})_{X}=|\mu(X)|$. It follows from Theorem 4.2 that $j$ induces an isomorphism $j_{X}: A O(\mathscr{A})_{X} \rightarrow W(\mathscr{A})_{X}$ for all $X \in L$. Thus it suffices to show that $\operatorname{dim} A O(\mathscr{A})_{X}=|\mu(X)|$. Since $A O(\mathscr{A})_{X}=A O\left(\mathscr{A}_{X}\right)_{X}$, we may assume that $X=\cap \mathscr{A}$ is the maximal element of $L(\mathscr{A})$. Choose coordinates so that $X=\left\{x_{1}=\cdots=x_{m}=0\right\}$. Suppose $\mathscr{B} \subset \mathscr{A}$ is independent and $\cap \mathscr{B}$ $=X$. Then $\mathscr{B}=\left\{H_{1}, \ldots, H_{m}\right\}$ and $d \alpha_{H_{1}} \wedge \cdots \wedge d \alpha_{H_{m}}$ is a constant multiple of $d x_{1} \wedge \cdots \wedge d x_{m}$. Recall the graded $\mathbf{K}$-algebra $R(\mathscr{A})$ generated by 1 and $d \alpha_{H} / \alpha_{H}$. It follows that multiplication by $d x_{1} \wedge \cdots \wedge d x_{m}$ induces an isomorphism $A O(\mathscr{A})_{X} \simeq R(\mathscr{A})^{m}$. It was shown in [6] (see also [7, 3.129]) that $\operatorname{dim} R(\mathscr{A})^{m}=$ $|\mu(X)|$ 
COROLlary 4.4. We have

$$
\operatorname{dim} W(\mathscr{A})=\operatorname{dim} U(\mathscr{A})=\operatorname{dim} A O(\mathscr{A})=\sum_{X \in L(\mathscr{A})}|\mu(X)| .
$$

When $\mathbf{K}=\mathbf{R}$, this number equals the number of chambers of $M(\mathscr{A})$.

Proof. The first part is by Theorem 4.3 and the fact that $(-1)^{r(X)} \mu(X)=$ $|\mu(X)|$, see [7, 2.47]. When $\mathbf{K}=\mathbf{R}$, connected components are called chambers. The second part follows from Zaslavsky's theorem [8].

\section{NBC bases}

In this section we construct explicit $\mathbf{K}$-bases for $A O(\mathscr{A}), U(\mathscr{A})$, and $W(\mathscr{A})$. These bases are in one-to-one correspondence with the set of NBCs (non-broken circuits).

Fix a total order on $\mathscr{A}$ by $\mathscr{A}=\left\{H_{1}, H_{2}, \ldots, H_{n}\right\}$. Recall that a subset $C$ of $\mathscr{A}$ is a circuit if it is a minimally dependent set. A subset $C$ of $\mathscr{A}$ is a broken circuit or a $B C$ if there exists a hyperplane $K \in \mathscr{A}$ satisfying $K<\min C$ so that the set $C \cup\{K\}$ is a circuit. A subset $T$ of $\mathscr{A}$ is called a non-broken circuit or an NBC if $T$ contains no broken circuit.

Lemma 5.1. If an independent subset $C$ of $\mathscr{A}$ contains a $B C$, then $Q(C)^{-1}$ is a linear combination of $\left\{Q(T)^{-1} \mid \cap T=\cap C, T\right.$ is an $\left.N B C\right\}$.

Proof. We may assume that $C=\left\{H_{i_{1}}, \ldots, H_{i_{m}}\right\}$ itself is a BC. Suppose that $T=\left\{H_{i_{0}}\right\} \cup C$ is a circuit and that $i_{0}<i_{1}<i_{2}<\cdots<i_{m}$. Let $T_{j}=T \backslash\left\{H_{i_{j}}\right\}$. This shows that $Q(C)^{-1}$ is a linear combination of $\left.\left\{Q\left(T_{j}\right)^{-1}\right\} \mid j=1, \ldots, m\right\}$. Note that $\cap C=\cap T=\cap T_{j}$ and we get the desired result.

For any subset $C=\left\{H_{i_{1}}, \ldots, H_{i_{m}}\right\}$ of $\mathscr{A}$, define

$$
\text { height }(C)=i_{1}+\cdots+i_{m}
$$

THEOREM 5.2. Let $X \in L$. The set

$$
N B C(\mathscr{A})_{X}=\left\{Q(C)^{-1} \mid C \text { in an } N B C \text { and } \cap C=X\right\}
$$

is a $\mathbf{K}$-basis for $A O(\mathscr{A})_{X}$. Therefore the set $\left\{Q(C)^{-1} \mid C\right.$ is an $\left.N B C\right\}$ is a $\mathbf{K}$-basis for $A O(\mathscr{A})$. 
Proof. It follows from [2],[4],[5] that the cardinality of the set

$$
\{C \mid C \text { is an } \mathrm{NBC} \text { and } \cap C=X\}
$$

is $|\mu(X)|$. We showed in the proof of Theorem 4.3 that $\operatorname{dim} A O(\mathscr{A})_{X}=|\mu(X)|$. Thus it suffices to show that $N B C(\mathscr{A})_{X}$ spans $A O(\mathscr{A})_{X}$. If not, there exists $C_{0}$ such that:

(1) $C_{0}$ is independent,

(2) $\cap C_{0}=X$,

(3) $Q\left(C_{0}\right)^{-1}$ is not spanned by elements of $N B C(\mathscr{A})_{X}$, and

(4) the height of $C_{0}$ is minimum among all subsets satisfying (1)-(3). Since $C_{0}$ is not an $N B C, Q\left(C_{0}\right)^{-1}$ is a linear combination of

$$
\left\{Q(T)^{-1} \mid T \text { is an NBC and } \cap T=X\right\}
$$

by Lemma 5.1 . By condition (4) and (3), this is a contradiction.

COROLlaRY 5.3. (i) The residue classes of the set $\left\{u_{C} \mid C\right.$ is an NBC\} give a $\mathbf{K}$-basis for $U(\mathscr{A})$ as a $\mathbf{K}$-vector space.

(ii) The residue classes of the set $\left\{Q(C)^{-1} \mid C\right.$ is an $\left.N B C\right\}$ give a $\mathbf{K}$-basis for $W(\mathscr{A})$ as a $\mathbf{K}$-vector space.

\section{REFERENCES}

[1] Aomoto, K., Hypergeometric functions, the past, today, and ......(from complex analytic view point), (in Japanese), Sügaku, 45 (1993), 208-220.

[2] Björner, A., On the homotopy of geometric lattices, Algebra Universalis, 14 (1982), $107-128$.

[3] Brieskorn, E., Sur les groupes de tresses, In: Séminaire Bourbaki 1971/72. Lecture Notes in Math., 317, Springer Verlag, 1973, pp. 21-44.

[4] Gelfand, I.M., Zelevinsky, A.V., Algebraic and combinatorial aspects of the general theory of hypergeometric functions, Funct. Anal. and Appl., 20 (1986), 183-197.

[ 5 ] Jambu, M., Terao, H., Arrangements of hyperplanes and broken circuits, In: Singularities. Contemporary Math., 90, Amer. Math. Soc., 1989. pp. 147-162.

[6] Orlik, P., Solomon, L., Combinatorics and topology of complements of hyperplanes, Invent. math. 56 (1980) 167-189.

[ 7 ] Orlik, P., Terao, H., Arrangements of hyperplanes, Grundlehren der math. Wiss., 300, Springer-Verlag, Berlin-Heidelberg-New York, 1992.

[8] Zaslavsky, T., Facing up to arrangements: Face-count formulas for partitions of space by hyperplanes, Memoirs Amer. Math. Soc., 154, 1975.

\section{Mathematics Department}

University of Wisconsin

Madison, WI 53706

U.S.A. 